Service social

\title{
Déterminisme ou liberté? Paradigmes scientifiques et intervention sociale
}

\section{Jean-Marc Dutrénit}

Volume 42, numéro 3, 1993

Perspectives théoriques

URI : https://id.erudit.org/iderudit/706633ar

DOI : https://doi.org/10.7202/706633ar

Aller au sommaire du numéro

Éditeur(s)

École de service social de l'Université Laval

ISSN

1708-1734 (numérique)

Découvrir la revue

Citer cet article

Dutrénit, J.-M. (1993). Déterminisme ou liberté? Paradigmes scientifiques et intervention sociale. Service social, 42(3), 103-136.

https://doi.org/10.7202/706633ar
Résumé de l'article

L'apparent enchevêtrement des théories de la société, de la personne et de l'intervention sociale peut être démêlé dès lors qu'on les rattache aux principaux paradigmes sources qui les inspirent. On découvre alors une très forte homologie de construction de ces théories à l'intérieur de chacun des quatre principaux paradigmes qui sont nés au cours du $\mathrm{XX}^{\mathrm{e}}$ siècle: fonctionnalisme (1920), habitus culturel (1960), ethnométhodo-logie (1965) et individualisme méthodologique (1980).

Parce que plusieurs de ces paradigmes, parfois contradictoires, sont souvent présents dans des séquences de travail social, leur mise à jour est essentielle pour comprendre les conséquences très concrètes des actes les plus quotidiens du travail social. 


\title{
Déterminisme ou liberté? Paradigmes scientifiques et intervention sociale
}

\author{
JEAN-MARC DUTRÉNIT \\ Professeur de sociologie \\ Université Charles-de Gaulle-Lille III, France
}

Aujourd'hui comme hier le travail social est tributaire des théories de la société et de la personne qui ont cours sur le marché des biens culturels. Aussi n'est-il pas inutile de faire le point sur ces théories, à une époque de crise des valeurs et des catégories de pensée des sciences humaines. On entendra par théories de la société les lois ou modèles de fonctionnement des sociétés humaines ou de leurs parties constitutives dès lors qu'elles forment une certaine cohérence. Ce sont évidemment la sociologie et dans une moindre mesure l'économie qui seront sollicitées pour définir ces théories. De même, on définira les théories de la personne comme les lois ou modèles de fonctionnement et de développement des individus à partir de la psychologie mais aussi partiellement des récentes découvertes de la biologie. Naturellement les théories de la socialisation et de l'identité seront particulièrement sollicitées du fait de leur situation articulatoire entre les décisions collectives de la société et les actes individuels des personnes. En ce qui concerne les théories de l'intervention sociale, on se référera indifféremment aux spécialistes des différentes sciences humaines qui ont écrit sur la question. 
Il eût été relativement facile de présenter successivement des listes de théories de la société puis des individus, puis du travail social en laissant au lecteur le soin de trouver les correspondances muettes entre les différentes théories des différents niveaux. Cependant, l'observation et I'analyse nous ont convaincu qu'un petit nombre de paradigmes opèrent de la même manière à travers les différents champs de la sociologie, de la psychologie et du travail social. C'est pourquoi, pour bien voir la filiation ou la parenté paradigmatique entre chaque champ théorique, il nous est apparu préférable de traiter les théories de la société, de la personne et du travail social en les classifiant au sein des quatre principaux paradigmes actuels en sciences humaines. Ajoutons que l'on entend par paradigme un ensemble de postulats et de méthodes $d^{\prime}$ analyse et de synthèse, donnant lieu à des écoles de pensée. Notre développement sera donc organisé selon les hypothèses qui suivent.

\section{HYPOTHÈSES}

1. Quatre grands paradigmes (conceptions de I'homme et de la société, méthodes et postulats) ordonnent la diversité des théories et des méthodes utilisées en sciences humaines. Deux types de postulats et de méthodes organisent ces paradigmes. Les uns postulent que la société pèse sur les individus et les détermine presque entièrement (Structuro-fonctionnalisme: SF et Habitus culturel: $\mathrm{HC}$ ), alors que les autres postulent au contraire que la société résulte de l'interaction et des stratégies des individus disposant d'une forte marge de liberté, organisant ainsi des systèmes multiples (Ethnométhodologie: EM et Individualisme méthodologique: IM). Deux types de méthodologie viennent recouper ces deux catégories. Le premier consiste à focaliser les recherches sur ce qu'on pourrait nommer la sémantique sociale en énonçant le sens des institutions, des fonctions, des stratégies d'acteurs (SF et EM). Le second privilégie la méthode expérimentale et ses dérivés pour décrire et décomposer les phénomènes à expliquer ( $\mathrm{HC}$ et IM). Ces quatre paradigmes représentent les principales orientations actuelles du monde des sciences humaines. Disons immédiatement qu'on ne prétend pas enfermer chaque auteur des sciences humaines dans l'un de ces quatre ensembles. Ceux-ci ne sont que l'expression de pôles ou de types-idéaux qui autorisent tous les dosages et mélanges intermédiaires. Pierre Ansart (1990) par exemple, dans un récent ouvrage, utilise une classification légèrement différente, mais $R$. Boudon (1982), H. Mendras (1989) ou C. Dubar (1991) ont produit des classements très voisins.

2. À chaque paradigme sociologique correspond une filière de conceptions de la personne et du travail social extrêmement typée et très 
cohérente. Aussi tenterons-nous dans cet article de décrire cette cohérence et la force d'entraînement de ces paradigmes à travers divers exemples, afin de proposer aux chercheurs comme aux praticiens une réflexion sur les implications pratiques des choix théoriques qui précèdent ou accompagnent leurs actes les plus quotidiens. Mais cela ne dépasserait guère l'exercice de style si l'on ne tentait pas en même temps une évaluation comparative de la plus ou moins grande pertinence de chaque paradigme, ce qui est évidemment essentiel autant pour les praticiens que pour les théoriciens. Ces exemples ne seront pas choisis arbitrairement, mais en fonction des études marquantes faites par les chercheurs se réclamant de l'un ou de l'autre des paradigmes. Mais on se gardera de prétendre à une présentation exhaustive des analyses représentatives de chaque paradigme. D'abord, elles abondent dans la littérature spécialisée et il serait impossible d'en rendre compte complètement, ensuite notre objectif est d'en constituer les types-idéaux afin d'en définir les usages possibles et leurs limites.

3. Les implications de ces filières sur le travail quotidien des travailleurs sociaux sont très puissantes dès lors qu'elles forment la base de leur idiosyncrasie. Comme en d'autres professions, ces choix préforment et prédisent largement le type d'acte professionnel.

4. Historiquement, il est certainement possible de trouver une succession ordonnée de ces quatre paradigmes. Le fonctionnalisme apparu autour de 1920 a dominé les théories de la société et de la personne jusqu'aux environs de 1960. À ce moment-là sont nés deux paradigmes de transition radicalement opposés I'un à l'autre: HC et EM. Le premier accusait les déterminismes fonctionnels, alors que le second les faisait voler en éclats. Enfin, vers 1980, I'individualisme méthodologique a permis de comprendre de façon opératoire les déterminations sociales comme résultantes des libertés individuelles et du pouvoir des acteurs. $L^{\prime}$ intervention sociale peut y trouver de solides points d'appui.

\section{LA FONDATION STRUCTURO-FONCTIONNALISTE}

\section{La société}

Plus personne ne se déclare fonctionnaliste en sociologie, et cependant le paradigme fonctionnaliste imprègne beaucoup d'analyses globales ou partielles de la société énoncées par des spécialistes comme par des profanes. Au cours de son existence le fonctionnalisme a pris plusieurs visages. Il repose $\mathrm{d}^{\prime}$ abord sur l'idée universelle de lien de cause à effet $Y$ phénomène à expliquer $=f(x l, x 2, \ldots x n$ variables explicatives $)$. $Y$ est " expliqué " comme fonction de $x$. Mais à son tour $Y$ sera la cause d'autres 
phénomènes «expliqués » de la même manière. L'intérêt de cette procédure est de mettre en rapport des phénomènes sociaux représentés par des variables avec d'autres phénomènes. Mais l'inconvénient est de faire disparaître ainsi le pouvoir et la liberté des hommes. À force de globaliser l'analyse, de prendre la tendance majoritaire ou la plus visible pour le tout, on oublie les variations internes, les oppositions, les contradictions. Par exemple, on pourra dire que la fonction de la famille est de reproduire la société telle qu'elle est de génération en génération. Mais ce faisant, on ignore le petit nombre de mariages qui ont lieu entre milieux sociaux différents, et on est en difficulté pour comprendre le changement et surtout les choix des acteurs. D'ailleurs, le problème du changement préoccupait peu le fondateur du fonctionnalisme. En effet, Malinowski cherchait davantage à comprendre la cohérence des sociétés polynésiennes qu'il avait sous les yeux en tant qu'administrateur de l'Empire britannique dans les années 20 qu'à percevoir des changements. Le fonctionnalisme s'est ainsi orienté vers l'anthropologie culturelle, cherchant à comprendre la diversité des sociétés et des réactions individuelles typiques dans chacune d'elles. L'exemple célèbre des personnalités plutôt agressives des Mundugumor opposées à la douceur générale des Arapech cité par Margaret Mead est devenu un des fondements de l'analyse fonctionnelle en sociologie: certains types d'institutions donneraient lieu à certains types de productions humaines de manière quasi automatique. À partir de 1940 Merton a enrichi l'analyse fonctionnelle pour distinguer les fonctions apparentes et les fonctions latentes sur divers objets. En outre, on a pu dire qu'il y avait chez Marx et chez Durkheim de nombreux éléments fonctionnalistes cependant que des théoriciens contemporains comme Davis et Moore ont tenté d'élaborer une théorie fonctionnelle de la division du travail.

L'analyse marxiste de la société et de ses différents stades (les modes de production asiatique, antique, féodal, capitaliste, socialiste, communiste) a été considérée à juste titre comme un système fonctionnel où chaque élément de société jouait son rôle, y compris les classes sociales dans leurs luttes et les révolutions qui en découlaient parfois. Pour prendre un exemple moins global encore chez Marx, "l'armée de réserve du capital » constituée par le chômage avait pour fonction de faire baisser les salaires, mais il a bien fallu voir par la suite qu'elle fait baisser aussi les profits et la croissance.

L'analyse durkheimienne de la division du travail peut être considérée également comme fonctionnaliste. Sociétés segmentaires et sociétés organiques, rôle des corporations et des divers groupes sociaux assumant toute une série de fonctions dans ces cadres généraux décrivent bien des systèmes cohérents dans lesquels « un fait social s'explique par un autre 
fait social ", comme le stipulait l'auteur des Règles de la méthode sociologique. Dans une synthèse encore actuelle, Davis et Moore ont réalisé une analyse fonctionnaliste de la division du travail, celle-ci engendrant des fonctions professionnelles diverses et de ce fait inégalement rémunérées. L'accès à ces rémunérations donne lieu à une concurrence qui trouve sa solution dans la conquête de compétences différentes qui ellesmêmes viendront renouveler la division du travail. On est là aussi devant un système cohérent et séduisant. Mais outre une forte tendance à en rester aux significations des phénomènes sans aller jusqu'à la vérification expérimentale, le fonctionnalisme doit répondre à plusieurs critiques fondamentales. D'abord celle de Claude Lévi-Strauss (1960): « dire que certaines choses ont une fonction dans la société est un truisme, mais dire que tout fonctionne est une absurdité ». Ensuite, il est difficile d'apporter la preuve que tel élément assure telle fonction et pas une autre, surtout lorsqu'il s'agit de fonction latente. Enfin, Olson (1978) a démontré que I'on fait une erreur cuisante en considérant que les sociétés évoluent par les mouvements d'une prétendue conscience collective se conduisant implicitement comme une conscience individuelle. Même les conceptions durkheimiennes de l'arrangement évolutif sous la poussée de nombreux groupes de pression - eux aussi analysés implicitement comme des acteurs individuels - tombent sous le coup de cette critique dont on verra la démonstration plus détaillée dans le paradigme de I'IM.

\section{La personne}

Sur le plan des théories de la personne, le paradigme fonctionnaliste a essentiellement recherché les effets des structures sur les individus. À ce titre il est l'objet de critiques importantes bien synthétisées par C. Dubar (1991):

- Il considère la formation de l'enfant sur le modèle du dressage ou selon le schéma du conditionnement qui impliquent l'assimilation précoce et inconsciente de schèmes corporels et d'attitudes culturelles qui sont censées déterminer ses conduites futures. Ce paradigme de la socialisation-conditionnement fait de l'individu socialisé une sorte d'automate déterminé ou programmé par ses expériences passées et non un acteur libre de ses choix et responsable de ses actes;

- il privilégie les expériences de la petite enfance et les « disciplines de base » imposées par la culture du groupe social d'origine: les premières relations, inconscientes ou refoulées, marquent l'individu plus que les suivantes et constituent des types de personnalité 
exprimant la culture du groupe d'origine. Ce paradigme «psychanalytique » imprègne plus ou moins toutes les versions de l'approche culturelle-fonctionnelle et fait de l'adulte socialisé un produit du complexe parental dont il est issu.

Cette critique est fondée sur l'analyse du courant de l'anthropologie culturelle: écho lointain et transfiguré de la théorie du conditionnement de Pavlov, il est construit au cours des années 30 par des auteurs comme Kardiner (1939), Ruth Benedict (1935), Linton (1936). Ces auteurs considèrent en effet que chaque culture fabrique des modèles qui se différencieraient tant de la nature humaine que celle-ci n'existerait pas. Or, comme le note également Dubar (1991), des travaux d'anthropologie cognitive (Dougherty, 1985) et de psychologie transculturelle (Warren, 1980) ont renouvelé la question des " universaux cognitifs » et ont montré que des comportements très divers, reliés à des formes très différentes d'apprentissage, pouvaient renvoyer aux mêmes processus cognitifs élémentaires (catégorisation, généralisation, différenciation, résolution de problèmes, etc.). Ce qui revient à dire que les travaux de Piaget ont probablement une valeur universelle. On est loin de la nature culturelle de l'individu.

Mais le paradigme fonctionnaliste a été encore largement utilisé dans les théories de la personne produites par des théoriciens classiquement fonctionnalistes comme Parsons (1955) et Merton (1950), des marxistes comme Sève ou des sociobiologistes comme Wilson. Pour Parsons la personne se constitue à l'intersection du biologique et du social par un processus de socialisation qui doit son originalité à une intégration de la psychanalyse selon Freud, de la psychologie des petites groupes selon Bales (1955) et du système social LIGA dont il est l'inventeur. La stabilité normative (désignée par L comme Latence), nécessaire au système social, est apprise au cours de la crise orale qui fait alterner dépendance et permissivité. L'intégration I des acteurs dans le système se fait ensuite par la phase œdipienne avec un attachement amoureux à divers supports. La fonction de définition des buts ( $\mathrm{G}$ comme goal-attainment) est assimilée au cours de l'adolescence en passant par un déni de réciprocité avant de déboucher sur la fonction d'adaptation (A) qui est l'âge de la maturité et de la manipulation des sanctions. Au fond, Parsons développe dans la société actuelle une bonne partie des théories énoncées par les culturalistes à propos des sociétés archaïques, à ceci près que l'action est moins prédéfinie dans la théorie parsonnienne que dans celle des culturalistes. Merton (1950) développe un peu plus encore les degrés de liberté de l'individu en parlant de socialisation anticipatrice: un individu peut apprendre et intérioriser les valeurs d'un groupe auquel il aimerait appartenir, et Merton le nomme groupe de référence. C'est un supplément de liberté dans le fonctionnalisme. 
En France où le fonctionnalisme marxiste a occupé une place de choix qui a laissé des traces, il faut mentionner la tentative de Lucien Sève (1970) pour plaider que la personnalité est engrenée dans la structure sociale de classe et résulterait de ce fait des parts respectives de son emploi du temps dans l'utilisation ou le développement de ses capacités de tous ordres, soit dans le travail abstrait (salariat), soit dans le travail concret (qui revient à l'individu). Conception intéressante par la notion de capacités plus ou moins développées, mais très fonctionnaliste au sens où I'initiative individuelle $n$ 'est guère envisagée que sous forme immergée dans la lutte des classes. On doit enfin mentionner les dernières formes de fonctionnalisme dans les essais de la sociobiologie selon lesquels I'individu n'est plus qu'un transmetteur de gènes et ses comportements sont d'abord des messages biologiques de lutte pour le développement de l'espèce (Wilson, 1970). Au total, les théories fonctionnalistes de la personne ont pour ainsi dire gommé, évacué le pouvoir individuel et ce qui le fonde: libre arbitre, création normative et institutionnelle, stratégie d'acteur, etc. La personne est vue comme une pâte amorphe ayant peu ou pas voix au chapitre. La rançon de ce paradigme appliqué à la personne est un point aveugle: la responsabilité politique est revendiquée, notamment par le courant marxiste, mais en l'absence de théorie de liaison entre personne et évolution des institutions permettant de penser leur interaction, on ne sait jamais à qui elle doit s'appliquer ni comment elle doit être exercée. Aussi, I'apparition de leaders charismatiques exceptionnels qui forcent les événements (Jésus, Lénine, etc.) demeure parfaitement mystérieuse.

\section{L'intervention sociale}

Fortes des leçons de cette analyse, les théories de l'intervention sociale prennent du relief. On pourra ainsi considérer que le fonctionnalisme classique a donné lieu à des politiques sociales institutionnelles, centrées sur l'éducation en établissements pour enfants (IMP, foyers de l'Aide sociale à l'enfance), tandis que les adultes ne recevraient que la portion congrue sous forme d'un accompagnement sans espoir. Aux États-Unis, il semble bien que les hésitations et les malentendus de la Poverty War des années 60 aient été inspirés par le même type de sources. Symétriquement, et un peu plus tard, les fonctionnalistes marxistes se scandalisaient de cette "police des familles» (Donzelot, 1975; Chauvière, 1978) : la théorie du contrôle social commençait à déstabiliser le travail social et à s'ériger en contre-théorie de l'intervention, sans pour autant offrir une solution de rechange. Néanmoins, comme nous avons exprimé ailleurs (Dutrénit, 1989) de façon assez complète les théories de l'intervention sociale liées au marxisme et au fonctionnalisme, nous nous bornerons ici 
à un rappel de ses principaux aspects et à quelques compléments. Puisque la théorie fonctionnaliste de la socialisation privilégie l'enfance comme moule de l'adulte, on peut y voir la raison théorique principale au fort courant interventionniste du placement d'enfants en dehors de leur famille. En France comme ailleurs il a contribué à l'éclosion de nombreux établissements pour mineurs dans les années 1950-1970. En accord avec cette mouvance théorique, tous les degrés de liaison avec la psychanalyse se développaient dans les organismes de traitement. Dans l'attente de l'éclosion des sciences de l'éducation, le mouvement interventionniste consiste d'ailleurs en une éducation normative assez classique qui insiste sur la rupture nécessaire avec la famille d'origine.

À une époque où la formation permanente est encore balbutiante, le traitement des adultes est réduit à une aide ponctuelle et incertaine en milieu ouvert à part quelques asiles de nuit. Comme le chômage est infinitésimal, le phénomène est restreint. II y a à l'époque, bien sûr, l'aide aux migrants et au quart-monde, mais les théories hésitent entre un fonctionnalisme global (Gans, 1978) et un marxisme dur (Mury, 1972) aussi impuissants l'un que l'autre à sortir d'affaire les clients touchés. Le premier propose des regroupements associatifs afin de jouer sur des fonctions et les pouvoirs de groupe d'appartenance, de référence et de rejet (Alinski, 1960) ou renvoie l'issue à une époque lointaine lorsque la pauvreté sera devenue dysfonctionnelle pour les riches, le second ne peut offrir que I'intégration dans la lutte des classes ou le mépris dévolu au sous-prolétariat. Sur le terrain la Poverty War avait été un échec retentissant (Moynihan, 1970), et la théorie de l'intervention sociale se trouvait par conséquent dans une sorte d'impasse molle. Une forme plus récente du fonctionnalisme en théorie de l'intervention est survenue avec la tentative de modèle d'évaluation dit clinique lancé par Anne-Marie Favard (1986). D'après son appréciation, les acteurs du travail social prétendent fonder leur action sur la clinique, mais en réalité les fonctions socio-judiciaires des établissements reprennent vite le dessus pour gérer la clientèle selon des procédés de la faute, de la peine et de la sanction, puisqu'ils ont force de loi. L'auteur généralise son propos, basé sur l'observation du circuit des mineurs délinquants, à l'ensemble du travail social, car d'après elle la clinique est trop balbutiante pour prendre un leadership quelconque. Hypothèse fonctionnaliste et foucaldienne $s^{\prime} i l$ en est: la fonction de la clinique est de masquer le vrai visage répressif de l'appareil du travail social. Une avancée significative allait venir de deux paradigmes nouveaux bien que radicalement opposés l'un à l'autre: celui de l'habitus culturel et celui de l'ethnométhodologie. 


\section{DEUX PARADIGMES DE TRANSITION : L'HABITUS CULTUREL ET L'ETHNOMÉTHODOLOGIE}

\section{L'habitus culturel}

Le paradigme de l'habitus culturel est d'abord une sociographie, liée au nom de son créateur Pierre Bourdieu. Comme le fonctionnalisme, il considère que la société détermine l'individu dans l'enfance d'abord, mais aussi dans l'âge adulte, comme dans le marxisme. Mais ce $n^{\prime}$ est pas la psychanalyse qui explique le relais société-individu, c'est l'habitus. Pour le démontrer les émules de ce paradigme utilisent beaucoup les statistiques, et singulièrement celles qui portent sur l'éducation.

Dans leur premier ouvrage sur la question, Bourdieu et Passeron (1960) montrent ainsi que les étudiants de la fin des années 50 sont en écrasante majorité issus de familles cultivées depuis une et souvent deux générations. Ils trouvent à l'école puis à l'université ce qu'ils avaient déjà chez eux: la culture scolaire. Dans les œuvres postérieures comme La Reproduction et La Distinction, Bourdieu assigne à la structure scolaire une fonction de reproduction de la structure de classe de la société. II y a selon Bourdieu "collusion objective" entre l'école et la bourgeoisie pour conserver à celle-ci les meilleures places que celle-là permet d'obtenir et de légitimer. Même si aujourd'hui les classes populaires accèdent à l'université, I'auteur explique qu'elles sont « reléguées » dans les disciplines aux débouchés aléatoires, alors que la bourgeoisie place ses héritiers dans les grandes écoles et les écoles de gestion. Si cette analyse correspond à une partie de la réalité, elle se heurte à une objection de taille. Si les structures de l'enseignement assurent la reproduction de la société sur un mode conservateur, comment expliquer la transformation radicale de la société française sur le plan des catégories sociales auxquelles I'école donne accès, comme en témoignent les statistiques de I'INSEE (Djider, 1990) ? Car s'il est vrai que certains changements renouvellent des différences et des distinctions, il est également vrai qu'une société dont $45 \%$ d'une classe d'âge a atteint le niveau du baccalauréat ne se conduit pas comme une société ayant $3 \%$ ou $4 \%$ de bacheliers (chiffres des années 30).

Au fond, le paradigme de l'habitus culturel est un héritier assez direct quoique dissimulé, précieux et très contorsionné d'un fonctionnalisme marxiste. Il a hérité des classes, des structures, de la reproduction mais pas de la révolution. C'est un révolutionarisme stationnaire, plus proche de la révolte que de la fécondité sociale, plus enclin, semble-t-il, à satisfaire la contrition des chers intellectuels de gauche qu'à trouver des 
solutions et des remèdes aux "collusions » qu'il dénonce. C'est une sociographie convenable et parfois jubilatoire, mais qui ne débouche sur aucune perspective et ne rend compte que d'une partie des statistiques, celles qui justifient la reproduction par opposition à celles qui montrent la transformation.

La théorie de la personne qui permet et justifie ces conceptions n'est guère différente de celle du paradigme fonctionnaliste. Comme le dit Padioleau (1986) de manière imagée, il s'agit de l'Homo Sociologicus éponge qui, comme son nom l'indique, absorbe sans discernement tout ce qui passe à sa portée. Pris au piège de ses hypothèses déterministes, ce paradigme développe une théorie extrêmement restreinte de la personne qu'on pourrait résumer dans une formule "Dis-moi la catégorie socioprofessionnelle (CSP) de tes parents et je te dirai ton avenir ». En termes savants, on dira avec F. Heran (1987) que le concept d'habitus, clé de cette théorie, "s'inscrit dans la lignée de ces concepts intermédiaires que nombre d'auteurs ont tenté d'imposer entre le subjectif et l'objectif, l'individuel et l'institutionnel: ainsi aliénation, attitude ou ethos. [...] Par la médiation de l'habitus, le dépôt des expériences passées se convertit en disposition pour l'avenir, l'habitudo se fait habilitas. » Le fondement de la théorie de la personne exprimé là est finalement une "force de commutation ou d'activation [...] qui rendrait caducs les antagonismes entre spontanéisme et mécanisme, liberté et déterminisme, objet et sujet, acteur et structure, individu et société, etc. ». Or, rappelle F. Heran, l'opération consistant à surmonter ces dichotomies est une vieille tradition philosophique, de Platon à Husserl, mais elle n'est pas pour autant explicative. Les mythes eux-mêmes utilisent cette procédure. Cependant, les utilisateurs du paradigme de $\mathrm{I}^{\prime} \mathrm{HC}$ réduisent ce pouvoir de commutation à une perpétuation des groupes sociaux et de la société dans le même état. De ce fait la personne ne représente guère qu'un intermédiaire structurel aux initiatives réduites par "l'intériorisation des conditions objectives» de sa position de classe.

La théorie de l'intervention sociale, parente de ce paradigme, pas très cohérente sur le plan logique, est assez décevante sur le plan de l'action. Elle consiste d'abord à dénoncer les structures et les institutions productrices d'habitus. Les travaux de ce genre ne manquent pas (Lascoumes, Verdès-Leroux, Bobrof et Luccioni, Barbier, Chamboredon, Chauvière), que l'on a analysés ailleurs (Dutrénit; 1989). On se contentera ici de les résumer en quelques lignes. Pour Lascoumes la cancérisation du travail social est un instrument du contrôle social, c'est-à-dire la reproduction de la société de classe et de la domination capitaliste. Pour Verdès-Leroux, les travailleurs sociaux par leurs enquêtes invalident leur clientèle (pauvre, malade, etc.), afin de mieux "briser » ce qui lui resterait de conscience de classe. Pour Barbier, les pièges institutionnels ne laissent au 
travailleur social d'autre choix que l'obéissance, l'adaptation secondaire ou la démission. Pour Chamboredon les agents de traitement de la délinquance juvénile font partie de la petite bourgeoisie au jugement étroit et strict, peu encline à comprendre ce genre de clientèle. Pour Chauvière enfin, le fait qu'une partie du dispositif de protection de l'enfance ait été construite sous le gouvernement de Vichy l'invalide et l'entache de défauts majeurs. Au terme de ces analyses, que reste-t-il pour l'intervention sociale des partisans de ce paradigme? À peu près rien, sinon impuissance et contrition. La responsabilité de la plupart des actes individuels se trouve éteinte par l'excuse de l'habitus contre lequel on ne pourra rien. Échec scolaire, délinquance, pauvreté, on n'en peut mais! C'est ainsi qu'est née la pratique rampante de "l'accompagnement » et de la lamentation stérile. C'est ainsi qu'est née aussi l'expérience de recrutement d'éducateurs spéciaux «étés chauds » dans les quartiers des banlieues à problèmes avec l'hypothèse que la proximité de leurs habitus culturels et de ceux de leurs futures ouailles serait un gage de réussite. Ce qui a évidemment été vérifié par le résultat contraire: plutôt davantage d'échecs. $C^{\prime}$ est ainsi que se sont développés des établissements-parkings, des stagesparkings et des écoles de travailleurs sociaux-parcomètres! Cercle vicieux de la pauvreté de l'échec et du travail social "babacool*» entretenu par le paradigme de l'habitus culturel déterminé-déterminant. Fascinés par des descriptions bien choisies et des exemples illustrant une théorie partiellement vraie, de nombreux travailleurs sociaux et responsables ont trop vite fait de la généraliser et d'interrompre nombre de leurs activités dès lors prises pour de l'activisme.

\section{L'ethnométhodologie}

À partir de 1960, et en concurrence ouverte avec le fonctionnalisme, puis I'habitus culturel, naissait le paradigme de l'ethnométhodologie, appelé aussi interactionnisme symbolique. À l'inverse de ceux-là, pour l'EM l'individu n'est plus la conséquence mais l'origine de la société et de ses institutions. Désordre et hasard retrouvent une place dans la construction des figures de société. Le déterminisme est inversé. Nous sommes des «sociologues à l'état pratique» selon la formule d'A. Schutz (1987). "Le réel est déjà décrit par les gens. Le langage ordinaire dit la réalité sociale, la décrit et la constitue en même temps ", comme l'écrit A. Coulon (1987). Reprenant la lecture de Durkheim, H. Garfinkel (1967), fondateur de ce nouveau paradigme, écrit que si «la réalité objective des faits sociaux est le principe fondamental de la sociologie», elle est également «en tant que

* babacool : terme d'argot intellectuel désignant en Europe une attitude soixante-huitarde, faite de laisser-aller affecté sur les plans ethique, intellectuel et vestimentaire. 
toute société est produite localement, naturellement organisée, réflexivement descriptible, accomplissement continu et pratique ».

On trouve dans la production sociologique récente des utilisations éclairantes de ce paradigme fécond. Par exemple, M. Dobry (1990) analyse les brusques changements de régime politique dans les périodes de crise selon trois étapes fondamentales. La première est une longue période de maturation au cours de laquelle les responsables ne croient plus aux valeurs officielles dominant la vie sociale, mais pendant laquelle, en même temps, ils sont solidaires parce qu'ils se croient coincés dans le jeu. La deuxième est celle de la multiplication des interactions pendant la crise révolutionnaire: la parcellisation des logiques organisationnelles est bousculée par l'événement, interrompant les calculs sur le possible et le probable; "ce qui est décisif est la visibilité de l'engagement des autres dans la mobilisation ». Que l'on considère mai 68 ou les nombreux épisodes de crises et révolutions en France ou dans d'autres pays, et tout récemment les pays de l'Est, M. Dobry attire notre attention sur " I'ampleur et la diversité des marchandages » de ces épisodes: I'essentiel se joue dans de véritables tests de position des uns vis-à-vis des autres. La troisième étape est celle $d^{\prime}$ 'un début de stabilisation des incertitudes fortes engendrées par la crise, en général autour d'un chef charismatique (Eltsine, Bonaparte, Lénine, de Gaulle, etc.). Nul miracle donc dans la naissance et le déroulement des crises, mais des interactions, des calculs et des marchandages.

Michel Crozier (1977) avait déjà analysé les mouvements lents des organisations comme résultant des négociations permanentes entre la règle officielle et la règle implicite avec lesquelles les acteurs jouent en permanence pour développer leurs stratégies, leurs alliances et obtenir des satisfactions au terme desquelles les institutions sont transformées progressivement. Dix ans plus tard, Renaud Sainsaulieu (1987) ajoutait que les conceptions de l'entreprise changent sous la poussée des acteurs dans les contingences du temps: "Profit, investissement, emploi, adaptations technologiques, développement local, métier et renouvellement professionnel, modes de vie et de temps au travail, formation qualifiante des adultes, culture et identités collectives, libertés syndicales et démocratiques sont autant d'objectifs assignés par les uns et les autres à l'entreprise, dès lors qu'elle compte pour beaucoup. » En même temps, l'entreprise comme institution offre aux acteurs la possibilité de modifier leurs modèles de comportement. Pour agir dans la crise, il ne suffit plus de se fier à des règles ni à des actions de masse conduites par des leaders. II est nécessaire de devenir plus créatif, de gérer sa formation continue, de participer à des mobilisations autour de défis commerciaux et techniques pouvant aller jusqu'à la gestion et au rachat d'entreprises. Les acteurs dans le siècle saisissent plus ou moins les occasions qui leur sont offertes, et ainsi se 
construit quotidiennement la société. Plus on approfondit les ressorts des événements, plus les déterminismes s'évanouissent.

Mais on doit dire également que l'ethnométhodologie a eu ses précurseurs. La notion de réciprocité comme "I'un des rocs de base» sur lesquels s'appuie la civilisation humaine avait émergé des observations de Marcel Mauss (1933) sur les gestes les plus quotidiens du don et du contre-don dans de nombreuses sociétés archaïques et modernes.

Dans une perspective plus modeste mais essentielle, Jean Kellerhals (1987), en Suisse, montrait que la vie quotidienne des familles s'organise selon des règles créées par les individus et que ces règles sont réparties aléatoirement dans les classes sociales. Il obtenait leur énoncé au moyen $d^{\prime}$ entretiens avec les familles leur demandant de dire elles-mêmes leur mode de fonctionnement sous forme $d^{\prime}$ une liste libre progressivement réduite à une dizaine d'éléments, ce qui est en accord parfait avec le paradigme de I'EM. Au même moment, en France, D. Bertaux-Wiame (1987) montrait que la mobilité sociale intergénérationnelle dépend autant des individus que de leur famille: "Les membres de chaque famille participent à la construction de la trajectoire familiale; et $c^{\prime}$ est de celle-ci en retour que dépend la pente de leur propre trajet individuel ». De cette remarquable recherche, on peut déduire plusieurs leçons. La présence $d^{\prime}$ 'un projet ou même d'une simple disposition (attitude, fonds biologique personnel...) à saisir les occasions est un élément moteur de mobilité sociale inter et intragénérationnelle. Dans le groupe familial, les conversations des femmes avec l'entourage apportent des éléments stimulants notables. Des familles analogues placées dans des situations analogues réagissent différemment, ce qui signifie une absence de déterminisme à ce niveau. Le projet ne se réduit pas, comme le prétend le paradigme de $\mathrm{I}^{\prime} \mathrm{HC}$, à une "intériorisation» de "l'extériorité objective ", car celle-ci demeure ignorée des acteurs. C'est la perception de l'extériorité qui est déterminante et qui résulte d'un travail de la subjectivité (fonctionnement cérébral complexe encore mal connu dans l'état actuel des recherches) dans lequel imagination, volonté, valeurs, identité personnelle ont leur part.

Dans une perspective voisine, Zaïhia Zeroulou (1988) montrait que la réussite scolaire des enfants maghrébins de familles immigrées en France dépend du projet familial d'intégration dans le pays d'accueil. Dans le champ proprement scolaire, les études de H. Mehan (1978) aux États-Unis avaient déjà montré que des formes d'interaction en classe, requises par les professeurs, doivent s'ajouter au contenu pédagogique pour qu'un élève soit déclaré compétent. Elles montraient aussi qu'aux tests et examens un fort degré de variation de l'aide arbitraire du testeur vient perturber les résultats de + à $-30 \%$ du score. De son côté, Erickson (1975) montrait qu'au cours des séances de conseils d'orientation aux 
élèves, la suite du cursus des études à accomplir était présentée soit comme une série d'obstacles insurmontables, soit comme un ensemble $d^{\prime}$ apprentissages normaux et faciles selon les sympathies et préjugés du conseiller pour l'élève. "La respiration dans le même rythme, des voix douces et harmonieuses, des gestes synchronisés entre les protagonistes jouent un rôle dans le choix de présentation.»

La société ne détermine pas la personne, mais est déterminée par un jeu complexe entre personnes, tel est l'enseignement du paradigme de $I^{\prime} E M$. Ce qui ne veut pas dire qu'elle échappe à toute détermination ni que ce jeu complexe soit sans règles et qu'on puisse faire $n^{\prime}$ importe quoi. Quelques règles de ce jeu ont été définies par l'IM que nous examinerons plus bas. Quant à la personne, voyons maintenant ce qu'en dit l'EM. Une chose est sûre dans ce paradigme, la personne est affranchie des déterminismes sociaux. Comme l'écrit Dubar (1991) qui a bien étudié la question, et dont on résumera ici le travail, elle résulte d'une "socialisation comme construction sociale de la réalité ", c'est-à-dire que l'individu emprunte au monde, en liberté, les divers matériaux de construction qui édifieront sa personne. À ce titre, il retient la distinction de Habermas (1981) entre trois types de médiations constructives du sujet: celle de l'interaction réciproque, celle de la représentation symbolique et celle du processus de travail. La dialectique de l'interaction aboutit à une reconnaissance réciproque au terme de laquelle: "Chacun est le même que l'autre dans ce en quoi il est opposé à lui. Se distinguer de l'autre c'est donc pour lui se poser comme le même que l'autre et $c^{\prime}$ est là précisément un connaître en cela [...] que son opposition lui apparaît tourner à l'identité pour lui-même, autrement dit qu'il se sait être lui-même dans cette façon de se regarder en l'autre. " Cette reconnaissance réciproque, productrice d'une identité est vue par Habermas non pas comme une donnée première, mais comme le résultat $d^{\prime}$ une pratique sociale. Il en va de même pour la dialectique de la représentation symbolique qui se construit à partir du lien entre les activités de travail et celles de communication ("agir communicationnel ", c'est-à-dire pouvoir, production des normes, légitimité, libération, réciprocité). Quant au travail, - «l'agir instrumental»-, il construit la part $d^{\prime}$ identité relevant des règles techniques et des habitudes de travail.

Mais l'EM de la personne a des ancêtres parmi lesquels on doit citer Max Weber (1920) qui distingue à la suite de Tönies la communauté (parents, enfants, frères, sœurs) et la société (le reste de la nation). La première est le siège de la construction de l'identité personnelle (valeurs, traditions, émotions, sentiment d'appartenance commune), la seconde celui de l'identité sociale (arrangement d'intérêts variés par contrat, droit, conventions, organisation rationnelle). Mais Weber (1946) a noté que la lente montée de la rationalisation de la vie sociale commencée à la 
Renaissance s'accélère dans tous les domaines et que par conséquent l'identité sociétaire dominera de plus en plus l'identité communautaire. Mais comme la société est divisée en secteurs relativement étanches les uns par rapport aux autres (économie, politique, juridique, militaire, éducatif, administratif, etc.), on aboutira à une diversification croissante des identités avec des degrés croissants de liberté dans leur construction.

George Herbert Mead (1933) a également beaucoup contribué à une conception libre, c'est-à-dire interactive, de la personne. On retrouve chez lui la construction dialectique du moi et d'autrui déjà rencontrée chez Habermas reprenant Hegel: " le fait premier est l'acte social qui implique l'interaction de différents organismes, c'est-à-dire l'adaptation réciproque de leurs conduites dans l'élaboration du processus social ». Autrement dit, s'il y a stimulus, il y a réponse focalisée mais libre. La construction de l'autonomie se fait par étapes. D'abord, l'enfant joue les rôles des adultes proches avec des doubles divers et organise de la sorte les réactions qu'il provoque chez les autres et qu'il provoque ainsi en luimême. La deuxième étape consistera à apprendre les règles en passant $d^{\prime}$ un type de jeu semi-collectif (les indiens par exemple, où chacun fait presque ce qu'il veut) à des jeux réglés comme le football: on adopte des règles et on joue des rôles et des stratégies. Enfin, dans l'étape suivante, il y a reconnaissance de l'individu par les autres en tant que membre d'un groupe, qui produit le «moi » identifié par autrui et le «je» qui joue des rôles dans le groupe et contribue à sa transformation. La liberté consiste alors pour la personne à emprunter tout le long de son existence des rôles chez autrui en les filtrant selon ses propres attitudes pour en sélectionner certains (bon gardien de but, fort en maths...).

Berger et Luckmann (1966) ont fait faire quelques pas de plus à cette théorie de la personne en distinguant la socialisation primaire et la socialisation secondaire. La première correspond au cycle classique décrit par G.H. Mead, mais avec une accentuation des éléments cognitifs présents dans le langage. La seconde décrit l'incorporation des savoirs spécialisés et de ce qui $s^{\prime} y$ rapporte comme valeurs issues du monde professionnel: image de soi, image du métier, conceptions de la vie en rapport avec le métier, etc. Cette deuxième socialisation détermine avec la première une infinité de cas de figure allant de l'accord parfait à l'opposition évidente. En cas de contradiction la socialisation secondaire peut prendre le pas et remplacer la primaire sous cinq conditions dont on empruntera le résumé à C. Dubar (1991) :

- une prise de distance de rôles incluant une disjonction entre identité réelle et identité virtuelle (Goffman, 1963);

- des techniques spéciales assurant une forte identification au futur rôle et un fort engagement personnel; 
- un processus institutionnel d'initiation permettant une transformation réelle de la "maison " de l'individu et une implication des socialisateurs dans le passage d'une «maison » à l'autre;

- l'action continue d'un "appareil de conversation » permettant de maintenir, modifier et reconstruire la réalité subjective incluant une " contre-définition de la réalité " (transformation du monde vécue par la modification du langage);

- l'existence d'une "structure de plausibilité », c'est-à-dire d'une institution médiatrice ( laboratoire de transformation») permettant la conservation d'une partie de l'identité ancienne au fur et à mesure de l'identification à des autrui significatifs nouveaux perçus comme légitimes.

Il va de soi qu'un monde en mutation comme le nôtre, et comme beaucoup d'autres à de multiples époques, offrira beaucoup d'occasions de socialisation secondaire et que de la réussite de celles-ci dépendra le degré d'intégration de la société. Renaud Sainsaulieu (1977) a montré l'importance de phénomènes de ce type à propos de l'apparition des techniciens comme groupe important dans les entreprises, et dans une moindre mesure à propos des conditions de travail de chaque métier qui transforme secondairement l'identité de chacun. Dubet (1988) de son côté montrait que les jeunes des banlieues en galère choisissent assez délibérément leurs modèles identificatoires (loubar, ouvrier, technicien ou cadre, galérien ou musicien, etc.) sans qu'on puisse définir des déterminismes de filiation. Enfin, dans le domaine neuro-biologique, les recherches actuelles recensées par J.-P. Changeux (1992) montrent I'extraordinaire plasticité du cerveau et ses fortes capacités d'adaptation.

Sur le plan des théories de l'intervention sociale, l'EM a nourri et nourrit encore un certain nombre de méthodes de réhabilitation de la personne. Le casework par exemple ou les théories de Rogers ont eu leur heure de gloire. Le courant des pédagogies actives a fait également ses preuves et les sciences de l'éducation ont développé un courant de recherches proche de ce paradigme. Un peu plus tard la méthode PASS, très liée au mouvement de désinstitutionnalisation, a fait d'assez nombreux adeptes. Mais les aspects non élucidés de la personne dans les théories explorées ci-dessus, et inspirant ces types d'intervention, feront planer une part de mystère, de doute et d'approximation dans leur théorisation. Cette part non élucidée concerne au fond les capacités de l'individu et leur développement, qui seront mieux explorées par le courant expérimentaliste et cognitiviste postérieur, rattaché au paradigme de I'IM. En attendant, la connaissance des degrés de liberté individuelle dans I'interaction supposait évidemment d'offrir au client un minimum d'ouvertures et de possibilités d'inventer sa propre route, soit à partir de modèles 
externes, soit à partir du renvoi en miroir de sa propre image. À l'inverse des paradigmes précédents on compte sur la coopération du client, on la recherche, on la suscite. Le casework (aide psycho-sociale individualisée) selon Hunt et Kogan (1950) et Du Ranquet (1983) va jusqu'à établir un contrat entre le travailleur social et le client. La théorie rogérienne de l'intervention (Rogers, 1950 et plus tard) s'applique aux individus soit en face à face, soit en groupe. II s'agit d'aider les personnes à trouver une congruence maximale entre le dire et le faire, afin d'accéder à un accord harmonieux entre le moi idéal et le moi perçu. Pour cela, le thérapeute doit trouver la demande profonde du client, derrière ses mots, et la lui exprimer en miroir, autant que possible avec les mêmes mots. La découverte de soi-même précède ainsi une évolution future. Lorsque cela se déroule à l'intérieur d'un groupe, ce dernier se charge de la tâche avec plus ou moins de chaleur ou de violence, mais le plus souvent avec une grande efficacité. On postule que tout est possible, on cherche même à soigner la maladie mentale avec la psychothérapie. La dérive de la "client-centered therapy " commence à travers de nombreux épigones comme Max Pagès et $V$. De Gaulejac. Elle va se dégrader en non-directivité en de nombreux sites. La "relation » devient une sorte de panacée qui finit par perdre tout sens dès lors qu'elle ne s'inscrit pas dans une perspective, un contexte ou un parcours définis. Nous avons décrit de tels errements de la pratique éducative dans le chapitre "Des organisation incertaines » de notre livre de 1989 et il serait superflu de développer ce point.

Cependant, la pédagogie continuait ses recherches et l'école nouvelle de Célestin Freinet, en France du moins, faisait des adeptes. Il s'agit $d^{\prime}$ une pédagogie active très en vogue dans les institutions de l'enfance inadaptée, au cours des années soixante, qui sollicite la participation de l'enfant (coopérative de classe, textes libres, imprimerie, classe correspondante avec qui on échange les textes, etc.) Elle se situe parfaitement dans la mouvance de I'EM: prépondérance de l'individu qui renverse les traditions de la société, et allergie à la mesure chiffrée. Sans le rattacher à ce paradigme, il faut citer Guy Palmade (1976) qui, faisant une recension des pédagogies de l'époque, remarquait la meilleure performance, statistiquement significative, des méthodes actives pour les élèves les moins doués et moyens, alors qu'elles étaient identiques à celles des méthodes traditionnelles (ex cathedra) pour les élèves d'intelligence supérieure à la moyenne.

De leur côté, dès 1963 Wolfensberger et Glenn (1975) inscrivaient leur souci de briser le poids oppressant de l'institution sur l'individu en créant un système d'évaluation, le PASS, (Programme d'analyse des systèmes de service), mettant en lumière le degré de respect des droits et des libertés des clients que manifeste l'établissement. On peut certainement 
y voir une réaction contre le paradigme fonctionnaliste installant les handicapés dans des institutions. Une fois lancées les évaluations par le PASS, I'école de pensée correspondante a mis en marche un assez fort mouvement de désinstitutionnalisation. Les hypothèses de base consistent à dire que les handicapés sont malheureux et ne développent guère de capacités en institutions, et que celles-ci coûtent plus cher que des petits foyers pour deux à trois handicapés (Flynn, 1985).

Cet air de liberté dans I'intervention a beaucoup compté en France à partir de 1968. C'est ainsi que Donzelot a pu parler de «flottaison des images " des figures partenariales ou familiales organisée par les psychologues, notamment au cours de leur écoute des problèmes des clients. Dans un contexte d'incertitude de valeurs, cette méthode douce permet de faire évoluer les prises de position des partenaires - psys inclus - avec un minimum de contrainte et en quelque sorte au gré du vent. Seulement on reste dans le flou et le provisoire en permanence: on se contente de remplacer une incertitude par une autre et on recommence.

Une conception très libre de l'intervention sociale et de l'absence de fatalité héritée socialement a été élaborée récemment par Roger Dhuyme [....] au cours d'une enquête sur la reproduction du placement à l'Aide sociale à l'enfance. Contrairement à toute attente, seulement $5 \%$ des enfants placés en foyer ont eu des parents qui avaient eux-mêmes été placés pendant leur enfance. Cette rupture dans l'héritage des violences éducatives a fait du bruit dans le Landernau du travail social français, provoquant des dénégations véhémentes, mais aussi des inspirations: certains services ont mis en place avec succès des traitements $d^{\prime}$ éradication de la violence, comme celui de l'UDAF des Bouches-du-Rhône "Vivre sans violence». En conclusion, on pourrait dire que I'EM a beaucoup contribué à la réintégration de la liberté dans les théories de l'intervention, mais qu'il n'a pas eu assez d'exigence en précision sur le plan de I'usage et des conséquences de cette liberté dans le traitement social. L'IM allait y pourvoir quelque temps après.

\section{UN PARADIGME OPÉRATIONNEL: L'INDIVIDUALISME MÉTHODOLOGIQUE (IM)}

\section{La société}

Comme l'explique R. Boudon (1982), la grande affaire de l'IM consiste à comprendre et à expliquer le passage d'un état social $P$ à un état $\mathrm{P}^{\prime}$ en faisant intervenir les millions de stratégies individuelles qui produisent des effets imprévus, appelés aussi contre-intuitifs ou pervers, à l'image de ce 
que les économistes ont analysé dans les phénomènes du marché. Parmi de nombreux exemples de ce type d'analyse, on peut citer celui de la baisse du revenu relatif à la fin des études supérieures en France, entre 1955 et 1975. Le nombre d'étudiants pendant ces années-là a été multiplié par cinq, à partir de milliers de décisions individuelles consistant à prolonger des études. Mais la rareté de la qualification supérieure diminuant, il en est résulté une diminution des rémunérations relatives, et pas seulement en Lettres ou en Arts: un sortant moyen de Polytechnique en 1955 pouvait espérer dix ou douze fois le salaire minimum de l'époque, alors qu'aujourd'hui il s'agira de trois ou quatre fois ce salaire.

Un autre apport significatif de l'IM aux théories de la société a consisté à montrer de celle-ci la réalité du morcellement en multiples sousmarchés tirant chacun l'ensemble social à hue et à dia (Boudon, 1979). C'est La place du désordre (Boudon, 1984). Toute société est plastique et imprévisible globalement, mais prévisible dans ses parties constitutives ou certaines séquences à certaines conditions. Par exemple, dans L'inégalité des chances, Boudon $(1975,1985)$ encore montre que l'accès à l'enseignement supérieur d'un nombre croissant d'étudiants d'origine populaire ne modifiera pas de facto la structure sociale. D'autres éléments devront jouer pour cela, comme la structure de l'emploi et les échelles de rémunération.

Bien sûr, il faudra se garder de confondre I'IM avec une philosophie ou une idéologie individualiste qui mène souvent à l'isolement ou à la catastrophe. Comme le médecin qui examine un patient, détecte une maladie et préconise des mesures de prophylaxie pour la population, le sociologue utilisant I'IM analyse les stratégies individuelles et leur distribution pour comprendre et anticiper ce qui peut se produire. II s'agit dès lors d'annoncer à des groupes composés d'individus le résultat de cette anticipation. On est très loin d'une prise de position individualiste au sens classique du terme. C'est de méthode qu'il s'agit et non de finalité. L'IM n'est pas non plus fermé sur lui-même: il est possible que des éléments décrits par les autres paradigmes aient des influences sur les individus il serait absurde d'attribuer à I'IM la volonté de faire table rase -, mais ces influences sont toujours comprises à travers la médiation des individus qui sont responsables en dernière analyse, et qui sont le passage obligé - dans leurs actes et leurs stratégies - des vérifications expérimentales. Par exemple, Olson (1978) nous explique qu'on se simplifie trop la vie en attribuant à une prétendue conscience collective des caractères et des stratégies de type individuel. Ainsi, il est fréquent que des individus agissent exactement à l'encontre de leurs intérêts collectifs en tant que groupe ou catégorie sociale: on évite de participer à un syndicat, on s'abstient de manifester pour l'obtention d'un bien collectif, et en général on triche sur sa participation aux efforts pour produire ces biens collectifs (l'impôt entre autres 
choses). L'énorme faillite des sociétés socialistes, qui a commencé par la flânerie au travail et s'est poursuivie par un pillage organisé des biens collectifs, est un remarquable exemple de la validité de cette théorie. Le salaire restant le même quelle que soit la participation de chacun, il eût fallu beaucoup d'abnégation pour que tous fissent le maximum. Il y a cependant un moyen d'éviter les pièges de ce paradoxe d'Olson. Pour qu'un bien collectif soit obtenu grâce à la participation des individus, il faut que la collectivité concernée offre en retour à ces derniers des avantages individuels directs. La paix et la vie matérielle assurées par la communauté monastique ou la défense du médecin par l'American Medical Association contre les attaques en justice lorsqu'elles se présentent sont des moyens parmi d'autres de déjouer l'action de ce paradoxe de l'action collective. Lorsqu'une association, un syndicat ou un parti réunit ces conditions, il reçoit des adhésions.

Un autre aspect très fondamental de l'IM a consisté à démontrer la vraie nature et les composantes diverses de la structure inégalitaire des sociétés humaines. Nous en avions sondé la profondeur (Dutrénit, 1989) à la suite de Rawls (1987) et de Boudon (1979), mais Cherkaoui (1988) en a apporté une preuve supplémentaire intéressante. II a interrogé un échantillon de population sur son statut tel qu'il devrait être d'après la question suivante: "Supposons que I'on puisse classer les Français selon une échelle sociale allant de la catégorie la plus défavorisée (0) à la catégorie la plus favorisée (9). Dans quelle case vous placeriez-vous, au début de votre carrière, actuellement, et où pensez-vous que vous devriez être normalement? " Les réponses à chacune de ces trois questions présentent une distribution du statut fortement inégalitaire. Et I'auteur en conclut que: "Pour les individus, la société juste est foncièrement inégalitaire " et que "pour le statut revendiqué, l'inégalité est d'autant plus forte que la catégorie sociale (du répondant) est plus basse». Autrement dit, généralement, l'ambition de statut est liée à la situation où l'on se trouve, c'està-dire à la compétence sociale et professionnelle mise en œuvre. Voilà qui met les travailleurs sociaux devant un dilemme: ou bien on se contente $d^{\prime}$ aider les clients avec quelques secours et ils ne progresseront pas dans l'échelle sociale parce qu'ils s'estiment à leur juste place, ou bien on souhaite un progrès de cette clientèle, mais alors il est nécessaire de concevoir un modèle $d^{\prime}$ intervention basé sur une série d'apprentissages sociaux et professionnels programmés afin que cette clientèle puisse rehausser ses ambitions à partir d'une compétence acquise.

Les travaux de Marie Duru et Alain Mingat (1987) sur la stratégie des familles dans I'orientation des élèves indiquent la même direction de travail : lorsqu'on connaît le souhait d'orientation de la famille pour l'entrée d'un enfant en $4^{\mathrm{e}}$, on est en possession d'une prédiction aussi valable et 
pesante que celle qu'on peut tirer des résultats scolaires de l'enfant. La stratégie individuelle est donc essentielle. Toute intervention sociale devra en tenir compte. Des dizaines de travaux pourraient illustrer ces deux propositions. Citons pêle-mêle Peters et Waterman (1986) montrant qu'au fond les managers sont d'abord des administrateurs de valeurs morales déterminantes pour l'avenir de l'entreprise, March et Simon (1971) qui ont forgé le concept de rationalité limitée: on ne peut pas tout rationaliser, et surtout il y a des rationalités opposées, antagoniques, non conciliables, aussi légitimes les unes que les autres. Par exemple, les actionnaires d'une même entreprise peuvent opposer légitimement un projet de capitalisation pour les plus jeunes d'entre eux à la distribution de salaires importants pour les membres plus âgés. Mais il faudrait aussi rappeler les travaux de A. Toynbee (1975) qui montre que toute société ne parvient à survivre que si elle imagine en permanence des réponses aux défis renouvelés de l'environnement. Et cela n'est pas simple compte tenu du paradoxe $d^{\prime}$ Olson énoncé plus haut. Une bonne illustration de la validité de ces points de vue se trouve dans les plus récentes observations des pays en voie de développement. Citons Y. Platard (1991), A. Kabou (1990) et G. Sorman (1987), qui montrent très clairement combien l'incantation des défauts de la colonisation et les appels à l'aide extérieure ne peuvent remplacer les décisions locales des individus eux-mêmes. En élaborant des contrats progressifs de reconstruction par libération de l'initiative individuelle, en utilisant les ressources des nouvelles semences (et non en détruisant les stations agronomiques comme cela s'est produit en certains pays d'Afrique), en alphabétisant les femmes pour réduire la natalité, ces auteurs, et de nombreux autres, ont bien montré l'incroyable gisement de puissance que peut présenter l'individu à condition de le traiter dans les perspectives de I'IM que l'on vient de tracer brièvement.

Mais il serait injuste de terminer ce parcours sans citer l'un des pionniers de l'IM, Michel Crozier (1977), qui dès les années 50, en France, montrait comment l'acteur et le système combinent leurs énergies pour tout bloquer ou être créatifs selon les intérêts locaux en jeu à un moment donné. Dans ses plus récentes publications il explique fort bien comment " changer c'est changer la nature du jeu, développer à la fois de nouvelles capacités et de nouvelles règles ". Les unes sans les autres n'aboutissent qu'à des blocages et à des frustrations, sans compter les cercles vicieux de réciprocité négative qui en découlent. Les applications des résultats de ce paradigme pour l'intervention sociale, quelle que soit sa forme, sont si nombreuses et si fortes qu'elles favorisent un renouvellement profond des perspectives. Notre théorie de l'intervention élaborée dans Dutrénit (1989) en découlait déjà, et l'on verra plus bas que de nouveaux développements permettent $d^{\prime}$ aller encore plus loin. 


\section{La personne}

La théorie de la personne bâtie autour de ce paradigme de I'IM correspond aux formidables progrès de la psychologie cognitive expérimentale depuis 1970 auxquels on doit ajouter une récente contribution de $R$. Boudon (1990) à une sociologie cognitive qui donne une grande cohérence aux connaissances sur la personne aujourd'hui. D'après les résultats recensés par Doise, chaque personne élabore une stratégie d'accomplissement personnel ayant des conséquences sociales qu'elle anticipe mal ou peu:

1. elle tente d'acquérir ou de maintenir une identité sociale positive ;

2. cette identité positive est basée en bonne partie sur les comparaisons favorables qui peuvent être faites entre le groupe d'appartenance et certains autres groupes pertinents;

3. lorsque l'identité sociale est insatisfaisante, les personnes tenteront soit de quitter leur groupe pour rejoindre un groupe plus positif, soit de rendre leur groupe plus positif (Tajfel et Turner).

On voit bien ici que la personne agit avec son groupe d'appartenance, mais elle conserve de forts degrés de liberté comme le montrent les expériences sur la réactance. Lorsqu'un sujet est soumis à un stimulus léger (pour des achats, par exemple), l'influence est importante. Mais $\mathrm{si}$, au contraire, le stimulus est fort et insistant, il y a réactance, c'est-à-dire rejet pur et simple de l'incitation. Citons également les résultats à propos de la construction sociale de l'intelligence: le conflit socio-cognitif est producteur de progrès individuel à condition que soient opposés entre eux des individus de différents niveaux. On a ainsi constaté que les différences de niveaux scolaires atteints par des enfants de milieux sociaux différents s'atténuaient lorsque les moins privilégiés d'entre eux avaient eu l'occasion de coordonner leur action dans le cadre interactif de groupes de travail où ils rencontraient des enfants de milieux plus privilégiés.

Dans sa synthèse pour une théorie sociologique de l'identité, Dubar (1991) met au centre de la construction identitaire la dialectique entre une identité pour autrui, dans la mesure où autrui attribue à la personne une série de prédicats, estimations, considérations, et une identité pour soi correspondant à ce que l'individu ambitionne, produit, construit. Cela nous semble très proche du concept de statut social tel que nous l'avons défini (Dutrénit, 1989), auquel il faut ajouter maintenant la notion de compétence sociale permettant partiellement d'accéder à ce statut (Dutrénit,1992c). Posséder de la compétence sociale, c'est mettre en œuvre des contributions et recevoir en échange des rétributions dans un certain nombre de domaines de la vie sociale et de facteurs de la personnalité. Nous avons défini ces domaines et ces facteurs empiriquement par 
approximations successives avec de nombreux services sociaux dont nous dirigeons l'évaluation. Les domaines de compétence sociale pour un individu sont la gestion de sa formation, de son métier, de sa famille, de son budget, de sa santé, de son logement et de ses loisirs. Les facteurs de personnalité essentiels pour la compétence sociale sont la motivation, I'anticipation, la responsabilité, l'utilisation des acquis, l'image de soi, le pouvoir, le contrôle de soi. Mettre en œuvre de fortes contributions dans ces différents domaines et facteurs, et recevoir corrélativement de fortes rétributions permet de conquérir de la compétence sociale, donc à terme un statut social plus fort, c'est-à-dire ce que le travailleur social cherche pour sa clientèle: plus d'indépendance et plus d'autonomie.

Tout un courant des sciences de l'éducation a utilisé et développé les résultats de la psychologie cognitive (Postic et De Ketele, 1988; De Landesheere, 1988) de telle manière qu'ils puissent être utilisés par tout intervenant social. La conception de fond qui le sous-tend est de définir des situations maîtrisables par le formateur pour pouvoir les offrir aux personnes afin que celles-ci puissent acquérir les éléments de connaissance et les capacités qui les enrichiront, donc les transformeront. Citons quelques exemples de ces situations : répétition ou non des passages délicats d'un raisonnement sous plusieurs formes et à plusieurs moments ; qualité des rétroactions du professeur à partir des exercices de l'élève; prise en compte des acquis de ce dernier, etc. Et l'on aperçoit immédiatement que cela peut être mis en œuvre à propos de l'acquisition de la compétence sociale telle que nous l'avons définie. Cette stratégie est en prise directe sur I'IM car elle postule la liberté du formateur, celle de l'apprenant, et qu'elle utilise la mesure expérimentale pour asseoir ses résultats. Naturellement, il serait naîf de croire détenir là une nouvelle panacée. Maurice Cusson (1980) a montré par exemple qu'en prison environ un quart de la population, professionnalisée dans le crime, refusait délibérément toute participation à ce genre $d^{\prime}$ effort. On touche là une frontière entre deux rationalités opposées, bien illustrées par l'ouvrage de Bruce Jackson (1975).

Finalement, on peut certainement dire que l'identité personnelle se construit à partir d'une multiplicité d'influences dont chacun des paradigmes énoncés jusqu'ici analyse une partie. Comme le rappelle brillamment R. Boudon (1990), "la vérité n'est pas unique». Il est probable que les phénomènes repérés par les quatre paradigmes composent la personne comme un kaléidoscope compose une image. Mouvance perpétuelle ressemblant à celle des mouvements de nos dix milliards de neurones du cerveau dont les terminaisons sont en contact aléatoire pendant le sommeil. Merveilleuse image de la liberté intérieure: mes neurones $\mathrm{m}^{\prime}$ offrent des choix possibles, à moi d'agir (Changeux, 1992). Une fois un souvenir d'enfance $\mathrm{m}^{\prime}$ incitera à prendre telle orientation, une autre fois au 
contraire c'est un dialogue avec un collègue ou un client qui pourra $\mathrm{m}^{\prime}$ influencer. Ce sera encore la structure des possibles juridiques ou coutumiers à tel moment de ma vie, ou le pouvoir stratégique de mes partenaires qui me conduira à faire tel ou tel choix. Mon tempérament - probablement héritage génétique, et hasardeux comme tout héritage - viendra médiatiser tout cela. Les meilleurs biographes retrouvent alors les meilleurs historiens, les meilleurs sociologues et les meilleurs économistes, les meilleurs psychologues et les meilleurs neuro-physiologistes: I'homme est constitué de tant de déterminations que leur somme, ou mieux leur combinaison, $s^{\prime}$ apparente à ce qu'il est convenu de nommer le hasard. Il est libre. À lui d'utiliser cette liberté: il est responsable. Mais il se construira en fonction des disponibilités locales, selon les règles sociales et psychosociales minima repérées dans l'IM. Une théorie intégrée de l'intervention sociale devient possible. Il lui restera cependant à résoudre un problème fondamental: à partir de quel degré et de quelle qualité d'intervention l'individu décidera-t-il de "marcher », c'est-à-dire de modifier et d'enrichir ses valeurs, ses apprentissages, bref ce que $d^{\prime}$ autres nomment son habitus culturel? En considérant cet habitus non comme une donnée intangible, mais comme une somme structurée de compétences acquises (Dutrénit, 1992c), on peut espérer être sur la voie qui y conduit. À condition toutefois de bien considérer, comme R. Boudon (1990) I'a démontré, que chaque individu construit son idiosyncrasie à partir d'une « rationalité subjective». Elle « désigne ces raisons qui, bien qu'objectivement non fondées, sont cependant bel et bien des raisons ». Et, ajoute l'auteur, c'est tout le problème de la compréhension du sujet dans la société tel que le posait Max Weber: lorsque l'individu croit à des idées douteuses ou fausses, ce n'est pas parce que des causes inconscientes l'y poussent, mais parce qu'il a des raisons d'y croire. Nous en déduirons la nécessité de débusquer ces raisons bonnes mais incomplètes et d'offrir au sujet des raisons complémentaires pertinentes dans son univers personnel si l'on veut que l'intervention sociale soit opératoire.

\section{L'intervention sociale}

Mais très peu de chercheurs à notre connaissance ont travaillé la théorie de l'intervention sociale dans la perspective de I'IM, récente il faut bien le dire. Quels seraient ses caractères si l'on devait en tracer le portrait type?

1. Elle devrait d'abord comporter une stratégie d'analyse des "bonnes raisons " - au sens de Boudon - que la clientèle concernée a d'agir et de penser comme elle le fait. Des auteurs comme Carl Rogers (1955, 1965) ont illustré cette voie qui se démarque fortement de la psychana- 
lyse poursuivant des causes inconscientes. Au fond, il s'agit de pratiques socratiques du dialogue et des modernes analyses de contenu revues et corrigées par la recherche approfondie du raisonnement ordonné du sujet qui permet de retrouver les «bonnes raisons » implicites et explicites que celui-ci met en œuvre et qui le conduisent à croire en des idées « douteuses, fragiles ou fausses ». La définition de ce modèle pour chaque sujet est essentielle pour pouvoir comprendre ses stratégies et éventuellement tenter leur modification. Des points communs peuvent évidemment apparaître dans des groupes, ce qui facilite la tâche des interventions communautaires et de groupe. Tout le savoir accumulé par les autres paradigmes est utilisable à condition qu'il serve cette perspective de définition du raisonnement ordonné des sujets sur tel ou tel problème. Dans cette analyse des valeurs de l'individu on pourra s'appuyer en particulier sur la séquence de passages obligés décrits par Berger et Luckmann (1986) pour réussir une socialisation secondaire:

- disjonction entre identité réelle et virtuelle;

- action continue d'un appareil de conversation pour modifier et reconstruire le futur rôle.

2. Elle devrait ensuite comporter une stratégie de mise en œuvre médiatisée du conflit socio-cognitif tel que Doise (1978) le définit et que nous avons décrit ci-dessus. En faisant participer le sujet à des groupes différents de ceux qu'il fréquente dans son milieu d'origine, celui-ci devient capable d'intérioriser des capacités et une intelligence nouvelle des choses et du monde. Naturellement, il est nécessaire que cela ne s'éloigne pas trop des schèmes du sujet. Nous avons nommé cela dans d'autres travaux des "médiations relationnelles organisées ». Cela permet au sujet d'apercevoir de nouvelles perspectives pour son existence. Si la médiation est pertinente par rapport à son raisonnement ordonné, c'est-à-dire ni trop près ni trop loin, le conflit socio-cognitif est au maximum de son efficacité. Ces médiations devront en outre permettre une forte identification au futur rôle envisagé par le client dans sa socialisation secondaire et représenter une structure de plausibilité, $d$ 'institutions médiatrices accueillant l'individu pendant sa transformation (Berger et Luckmann, 1986).

3. Elle devrait aussi comporter une stratégie de contournement du paradoxe d'Olson examiné plus haut. Cela veut dire que toute intervention, qu'elle soit dite communautaire, de groupe, familiale ou individuelle, devra apporter aux individus des avantages immédiats, tangibles et individuels - fussent-ils identiques - aux protagonistes locaux. II est indispensable de trouver les stratégies gagnantes pour les acteurs en cause. Ici, les enseignements de M. Crozier (1978) s'avéreront utiles. Mais la technique du contrat, telle qu'elle est mise en œuvre dans le Revenu minimum 
$d^{\prime}$ insertion (RMI) français et plus encore dans le Workfare des États-Unis, constitue un élément essentiel d'une théorie de l'intervention basée sur $I^{\prime} I M$, parce qu'il garantit aux partenaires la recherche des conditions de gains mutuellement avantageux, conduisant à ce que nous avons appelé la Réciprocité positive (Dutrénit, 1989). Bien évidemment, il ne sera efficace que si les étapes de l'analyse des valeurs (rationalité subjective) du client et des médiations relationnelles organisées ont été parcourues convenablement et s'accompagnent de la recherche d'une identité positive du client (Doise, 1978). C'est aussi renouer avec l'interaction réciproque et équilibrée (Habernas, 1973) qui permet de transformer "I'agir instrumental » des normes de travail au même titre que d'autres éléments de I'organisation sociale.

4. Il faudra également qu'une théorie de l'intervention répondant aux critères de l'IM s'appuie sur des formations du client aux compétences sociales et professionnelles à côté des traditionnelles réhabilitations juridiques et financières. En effet, les travailleurs sociaux rencontrent la plupart du temps une clientèle déficitaire sur le plan de la formation scolaire et professionnelle, ce qui est connu (CNAF, CREDOC, Paugam), mais également faible dans ce que nous avons appelé, après d'autres (Salbreux, Tomkiewicz, Benoît), la compétence sociale, en donnant à cette notion le contenu précis exprimé plus haut. Les formations aux compétences sociales et professionnelles devront s'appuyer sur les trois éléments précédents (analyse des valeurs, médiations relationnelles organisées et contrat) pour être définies dans leurs orientations. Elles devront notamment tenir compte d'un diagnostic en compétence sociale (Dutrénit, 1992b). Un tel bilan peut comporter, pour être complet, le diagnostic en compétences sociales, en possessions sociales, en intégration sociale (Dutrénit, 1989, 1992b), un quotient intellectuel, une mesure du potentiel d'apprentissage de type "Learning Potential Assessment Device» (LPAD, Feuerstein et al., 1977). Ce point est très important, et Martine Delcroix-Collot (1992) a montré comment le LPAD permet " un ajustement des pratiques éducatives en termes de modalités, de quantité et de qualité d'investissements pour une planification pertinente de celles-ci ». Cela confirmerait s'il le fallait le bilan de nombreuses recherches en sciences de l'éducation, par Postic et De Ketele (1988), qui montrent que les dispositions intellectuelles pour recevoir les enseignements sont diverses pour chacun.

5. Ces apprentissages cognitifs ne seront efficaces qu'à condition d'offrir au client de nombreuses occasions d'exercice et de remédiation (De Landesheere, Feuerstein). Actuellement, une profusion de résultats peuvent être utilisés par les théoriciens et les praticiens de l'intervention sociale à propos des apprentissages. Citons-en quelques-uns. M. Bru (1992), qui a étudié la variété didactique dans la gestion des conditions 
d'apprentissage, montre à partir d'une expérimentation que les élèves ayant le plus de difficultés dans I'apprentissage de la lecture en $\mathrm{CP}^{*}$ sont ceux dont les maîtres présentent la plus grande variabilité didactique. Alors que si l'on met en relation l'action didactique, les conduites d'apprentissages et leurs résultats, on observe qu'aucun profil particulier d'enseignement ne peut être considéré comme la meilleure façon d'enseigner. Par conséquent, réduire la variabilité didactique de l'éducateur jouera très certainement un rôle déterminant, parmi d'autres, dans le succès de l'éducation. Ces exercices de remédiation pourront se faire individuellement et en petits groupes ad hoc (trois à sept personnes) avec un encadrement pius ou moins important selon les difficultés du client et le point de son parcours. Le travail en groupe est particulièrement important, car il peut être le siège du conflit socio-cognitif mentionné plus haut, de la constitution d'identité partielle, du développement imprévu de capacités pour atteindre les objectifs fixés (Doise, 1980).

Ajoutons que cet ensemble 1 à 5 représente ce que Berger et Luckmann (1986) appellent « un processus institutionnel d'initiation de l'individu permettant la transformation de sa "maison" originelle en une autre impliquant les résultants de sa socialisation secondaire ». Disons aussi que nous avons construit un progiciel de diagnostic du client sur les dimensions de compétence, d'intégration (insertion) et de possessions sociales ainsi que de mesure de l'intensité de travail social offerte au client. Son utilisation fonctionne maintenant dans plusieurs sites en France de telle sorte que ce progiciel n'est pas une évaluation externe du travail social, mais un instrument dans les mains de chaque travailleur social pour approfondir et systématiser son diagnostic et le traitement qu'il envisage. Ce progiciel a été expérimenté au Québec durant l'année 19931994.

Ajoutons encore que tout cela - éléments 1 à 5 - peut être planifié pour chaque client dans un programme ordonné à partir du diagnostic (Dutrénit, 1992b), soit le PEICS ou Projet éducatif individualisé en compétences sociales. Rappelons également qu'on peut le diviser en deux catégories: d'abord des rétributions du travail social adressées au client (points 1,2,3), puis des exigences adressées de même au client (4 et 5). Les évaluations que nous avons conduites jusqu'ici, celles qui existent et que nous avons analysées montrent qu'une forte intensité des éléments de l'intervention sociale que l'on vient d'énumérer est essentielle dans le progrès enregistré des clients qui en bénéficient. Parmi les évaluations que nous avons analysées, citons celle de Berk et Rauma (1982): pour les détenus sortant de prison, un service spécial de recherche de travail et le versement, pendant 13 semaines, d'une allocation de chômage

* Cours préparatoire - Enfants de 5 et 6 ans apprenant à lire. 
proportionnelle au travail effectué antérieurement en prison et cumulable avec des gains allant jusqu'à 150 \$ par semaine a permis d'observer une baisse statistiquement significative des récidives par rapport à ceux qui, n'ayant pas travaillé en prison, ne recevaient ni aide spéciale ni allocation. Chaque dollar investi dans l'opération a permis d'en économiser quatre du fait de la baisse des récidives. Cette évaluation est une preuve importante de l'efficacité de la dialectique exigence / rétribution dans un cadre théorique d'IM: les récidives diminuent dès lors que sont associées exigences fortes (travailler) et rétributions fortes (aide et allocations dépassant largement le pécule traditionnel et insuffisant accordé aux détenus libérés). Citons également J. Dumazedier, qui montre comment à Annecy, au foyer de réadaptation Le Logis, accueillant des jeunes cas sociaux et délinquants, la pratique de tous les sports est obligatoire pendant les six premiers mois de séjour, après quoi une spécialisation dans trois d'entre eux devient la règle incontournable. Dans cet exemple, les exigences d'apprentissage et de développement apportent en même temps les rétributions: révélation du corps (endomorphines cérébrales liées à l'effort physique), reconnaissance de son identité par autrui à travers les performances, rencontres organisées, puis contrats progressifs. Parmi les évaluations existantes, il faudrait en citer beaucoup d'autres. Le lecteur curieux pourra les trouver analysées dans Dutrénit (1989) et confrontées au concept opératoire exigences / rétributions. On devrait citer en outre les évaluations conduites en France par F. Le Poultier (1990) qui met en œuvre le paradigme de l'IM en conservant toutefois une description de l'intervention sociale si proche de celle des acteurs de terrain qu'elle conserve certaines redondances et notions floues comme "entretien avec le jeune, accompagnement, hébergement, etc. », toutes notions qui peuvent ou non contenir un ou plusieurs des cinq éléments essentiels relevés plus haut au titre de l'intervention idéale d'après l'IM.

Parmi les évaluations que nous avons conduites, citons-en deux. D'abord, dans trois établissements pour jeunes adultes handicapés mentaux légers - les CART (Centres d'adaptation et de réadaptation pour le travail) de Poitou-Charente -, la présence de fortes exigences associées à de fortes rétributions, mesurée par des descripteurs de notre progiciel évaluatif, entraîne un progrès statistiquement significatif des handicapés dans les domaines et les facteurs de compétence sociale cités plus haut (Dutrénit, 1989). Ensuite, l'audit-évaluation des sept clubs et équipes de Prévention de Seine-Maritime que nous avons conduit pour le Conseil général met en évidence la même chose: dès lors qu'une forte intensité des éléments exprimés plus haut comme étant l'intervention idéale $d^{\prime}$ après I'IM est présente, les performances de la clientèle sont bonnes pour elle et la société (autonomie, emploi, progrès en compétence sociale). 
On ne saurait terminer ce développement sur les rapports entre IM et intervention sociale sans dire quelques mots de la dialectique évaluation / intervention. Entraînée par les théories et les méthodes puissantes et performantes de I'IM , la théorie correspondante de I'intervention sociale consiste aussi en une dialectique permanente entre l'application des théories de la société et de l'individu élaborées par I'IM - c'est-à-dire l'organisation synergique des actes individuels dans le cadre $d^{\prime}$ 'une anticipation de leur effet collectif - et leur ajustement par évaluation systématique. C'est l'ère de l'évaluation et de la prévision (Dutrénit, 1992c) avec ses schémas directeurs locaux, ses évaluations ex ante, ses analyses et prévisions pour des caisses de sécurité sociale telles que nous avons pu en conduire. La théorie entraîne l'évaluation et réciproquement l'évaluation développe la théorie. C'est si vrai que le centre canadien de Boscoville a récemment infléchi son travail éducatif vers une aide éducative à la famille des jeunes délinquants qu'il héberge après avoir effectué une évaluation des anciens de l'établissement. Celle-ci faisait apparaître en effet que si les apprentissages de tous ordres restaient acquis pour la vie, l'intégration sociale laissait à désirer à cause des handicaps présents dans la famille d'origine (G. Trottier, 1992).

Naturellement, de nombreux obstacles se présenteront face à l'IM et à la théorie de l'intervention qui lui est liée, mais ses qualités et sa pertinence nous paraissent propres à lui assurer un succès grandissant.

\section{CONCLUSION}

En conclusion, nous pouvons prétendre avoir démontré nos hypothèses. Les paradigmes que nous avons définis tiennent à l'épreuve de l'inventaire des théories de la société, de la personne et de l'intervention sociale. Et il existe finalement autant de théories intégrées de la société, de la personne et de l'intervention sociale que de paradigmes, sachant que les implications de ces filières sont très puissantes sur le travail quotidien. On peut sur ces points faire deux remarques. Le développement suivi paraît démontrer que, contrairement à ce qu'on lit souvent en matière $d^{\prime}$ 'intervention sociale (Lecomte, 1991), il n'y a pas de différence entre paradigme de recherche et paradigme d'intervention.

En revanche, il y a très souvent des mélanges paradigmatiques tant sur le plan de la recherche que sur celui de l'intervention. Ensuite, il faut certainement se méfier du relativisme mettant en question le «mythe de l'objectivité ". S'il est certain que chaque paradigme a apporté une part de vérité, et que la vérité $n^{\prime}$ est pas unique parce qu'elle doit rendre compte de phénomènes aux facettes multiples, il est aussi certain qu'il 
existe des arguments ou des raisonnements faux ou incomplets et d'autres qui sont vrais et complets. Le problème devient alors d'en administrer la preuve. Sinon, on est propulsé dans un monde sans repères où toute parole en vaut une autre et dans lequel l'activité scientifique n'a plus de raison d'être. Les réflexions que suggère I'application de I'IM à l'intervention sociale nous incitent alors à situer l'intervenant comme être partiel mais non comme être "partial ", ainsi que le proposent Mayer et Ouellet (1991) après avoir éliminé "le mythe de l'objectivité ». Autrement, il ne $s^{\prime}$ orienterait guère vers la recherche de stratégies gagnantes pour les différents partenaires en présence et ferait très vite face à des effets imprévus souvent assez cuisants. Toutes les prises de parti ne se valent pas en intervention sociale, on l'a vu à plusieurs reprises dans cet article. Seules peuvent prétendre à la survie celles qui développent la réciprocité positive, c'est-à-dire qui se conforment volens nolens à l'une des plus fortes valeurs universelles des civilisations humaines. Et nous croyons avoir démontré que I'IM représente pour le moment le paradigme le plus approprié pour y parvenir.

\section{Références bibliographiques}

ALINSKI, S. (1960 [1975]). Manuel de I'animateur social. Paris: ESF.

ANSART, P. (1990). Les sociologies contemporaines. Paris: Seuil.

BALES et al. (1955). Family, socialization and interaction process. Glencoe: The Free Press.

BARBIER, P. (1973). "Une analyse institutionnelle du travail social ", Sociologie du travail, 1.

BENEDICT, R. (1935 [1950]). Échantillons de civilisation. Paris: Gallimard.

BENOÎT, O. (1965 [1990]). Statut dans l'entreprise et attitudes syndicales des ouvriers, dans R. BOUDON, L'art de se persuader. Paris: Seuil, p. V.

BENOÎT, O. (1990). "La recherche d'emploi (...) et qualification sociale », Sociologie du travail, 4.

BERGER, P.L. et T. LUCKMANN (1966 [1986]). La construction sociale de la réalité. Paris: Méridiens, p. 103.

BERK et RAUMA (1982). "Crime and poverty in California: some quasi experimental evidence », Soc. Science Research, 11, 318.

BERTAUX-WIAME, D. (1987). Le projet familial, Annales de Vaucresson, 1, 26.

BIENNALE DE L'ÉDUCATION ET DE LA FORMATION (1992). Résumé des communications. Paris: Association pour la promotion des recherches et des innovations en éducation et en formation (APRIEF).

BOBROF, J. et M. LUCCIONI (1975). La clientèle du travail social. Paris : ESF.

BOUDON, R. et P.F. LAZARSFELD (1971). Le vocabulaire des sciences sociales. Paris: Mouton. 
BOUDON, R. et F. BOURRICAUD (1982). Dictionnaire critique de la sociologie. Paris: PUF.

BOUDON, R. (1977). Effets pervers et ordre social. Paris: PUF.

BOUDON, R. (1979). La logique du social. Paris : Hachette.

BOUDON, R. (1984). La place du désordre. Paris: PUF.

BOUDON, R. (1985). L'inégalité des chances. Paris : Hachette.

BOUDON, R. (1986). L'idéologie. Paris : Fayard.

BoudON, R. (1990). L'art de se persuader des idées fausses, douteuses ou fragiles. Paris : Fayard.

BOURDieu, P. et J.-C. PASSERON (1960). Les héritiers: les étudiants et la culture. Paris : Minuit.

BOURDIEU, P. et J.-C. PASSERON (1970). La reproduction. Les fonctions du système d'enseignement. Paris: Minuit.

BOURDIEU, P. (1979). La distinction, Critique sociale du jugement. Paris: Minuit.

BRU, M. (1992). La variété didactique dans la gestion des conditions d'apprentissage. Biennale de l'éducation et de la formation. Paris-UNESCO.

CHAMBOREDON, J.-C. (1971). "La délinquance juvénile, Essai de construction d'objet ", Revue française de sociologie, XII : 335-377.

CHANGEUX, J.-P.(1992). La Recherche, juin.

ChAuVIÈRE, M. (1985). Enfance inadaptée: I'héritage de Vichy. Paris : Éd. ouvrières.

CHERKAOUI, M. (1988). "Mobilité sociale et équité », Revue française de sociologie, XXIX : 227-245.

COULON, A. (1987). L'ethnométhodologie. Paris: PUF, Que sais-je?

CREDOC (1991). Le RMI vu par ses allocataires, Commission nationale sur le RMI, 29 janvier 1991.

CROZIER, M. (1977). L'acteur et le système. Paris: Seuil.

CuSSON, M. (1983). Le contrôle social du crime. Paris : PUF.

DAUBIGNY-PRIANEZ, C. (1992). Psychopédagogie d'orientation psychanalytique pour la déficience intellectuelle. Biennale de l'éducation et de la formation. Paris-UNESCO.

DAVIS et MOORE (1945). "Some principles of stratification ", American Sociological Review, X, $2: 242-249$.

DE LANDESHEERE, V. (1988). Faire réussir, faire échouer. Paris: PUF.

DELCROIX-COLLOT, M. (1992). Ajuster nos pratiques éducatives par l'évaluation du potentiel d'apprentissage. Biennale de l'éducation et de la formation, Paris-UNESCO.

DIIDER, Z. (1990). Les catégories socioprofessionnelles. Données sociales, INSEE.

DOBRY, M. (1990). Sociologie des crises politiques. Paris : Presses de la Fondation nationale des sciences politiques (FNSP).

DOISE, W., J.-C. DESCHAMPS et G. MUGNY (1980). Psychologie sociale expérimentale. Paris : A. Colin.

DONZELOT, J. (1977). La police des familles. Paris: Minuit.

DOUGHERTY, J.W.D. (1985). Directions in cognitive anthropology. Chicago : Univ. of Illinois Press.

DU RANQUET, M. (1983). La recherche en casework. Toulouse: Privat et SaintHyacinthe: Edisem. 
DUBAR, C. (1991). La socialisation. Paris : A. Colin, p. 36-130.

DUBET, F. (1987). "Conduite marginale des jeunes et classes sociales», Revue française de sociologie, 2.

DUMAZEDIER, J. (1965). Loisirs et délinquance. Rapport pour le Ministre des Affaires sociales.

DuMAZEDIER, J. (1976). Société éducative et pouvoir culturel. Paris : Seuil.

DURKHEIM, E. (1902 [1960]). Les règles de la méthode sociologique. Paris: PUF.

DURKHEIM, E. (1967 [1993]). De la division du travail social. Paris : PUF.

DURU, M. et A. MINGAT (1987). "Facteurs institutionnels de la diversité des carrières scolaires", Revue française de sociologie, XXVIII : 3-16.

DUtRÉNIT, J.-M. (1989). Gestion et évaluation des services sociaux. Paris: Economica.

DUtRÉNIT, J.-M. (1992a). Perspectives méthodologiques pour l'évaluation des politiques sociales. Colloque des Caisses de retraite interprofessionnelles (Groupe CRI), Versailles.

DUTRÉNIT, J.-M. (1992b). "Évaluation du travail social, améliorations et développements récents", Travail social.

DUTRÉNIT, J.-M. (1992c). Habitus culturel ou compétence sociale. Biennale de I'éducation et de la formation, Paris-UNESCO, avril.

ERICKSON (1975). "Gatekeeping in the melting pot: interaction in the counseling encounters", Harvard Educational Review, 45, 1.

FAVARD, A.-M. (1986). "Méthodologie clinique de l'évaluation », dans CREAI de Poitou-Charentes, Outils d'évaluation en travail social. Angoulème: Multi.

FEUERSTEIN, R. et al. (1979). The dynamic assessment of retarded performers: the learning potential assessment device. Baltimore: University Park Press.

FLYNN, R.J. (1985). "Évaluation des programmes de désinstitutionnalisation", dans La désinstitutionnalisation : coût et incidence. Ottawa : Conseil canadien du développement social.

GANS (1972). "The positive functions of poverty », AJS, 78, 2: 275-289.

GARFINKEL, H. (1967 [1984]). Studies in Ethnomethodology. Englewood Cliffs, NJ: Prentice-Hall.

GeISSMAR, L.L. (1976). "Thirteen evaluative studies", dans E.J. MULLEN et J.R. DUMPSON, Evaluation of social intervention. Londres: J. Basch Publ.

GOFFMAN, E. (1963[1975]). Stigmate. Les usages sociaux des handicaps. Paris: Minuit.

GUINDON, J. (1972). Les étapes de la rééducation des jeunes délinquants... et des autres. Paris: Fleurus.

HABERMAS, J. (1981). Théorie de l'agir communicationnel, 2 t. Paris : Fayard.

HABERMAS, J. (1967 [1973]). "Travail et interaction. Remarques sur la philosophie de l'esprit de Hegel à léna », dans La technique et la science comme idéologie. Paris: Gallimard.

HERAN, F. (1987). "La seconde nature de I'habitus ", Revue française de sociologie, 3.

HUNT et KOGAN (1950). Measuring Results in Social Casework, N.Y. Family Serv. Assoc. of America.

JACKSON, B. (1975). Leurs prisons. Paris: Plon. 
KABOU, A. (1990). Et si l'Afrique refusait le développement ? Paris : L'Harmattan. KARDINER, A. (1939 [1969]). L'individu et sa société. Paris: Gallimard.

Kellerhals, J. et P.-Y. TROUtOT (1987). «Milieu social et types de familles: une approche interactive», Annales de Vaucresson, 1, 26.

LASCOUMES, P. (1977). Prévention et contrôle social. Genève: Médecine et Hygiène / Masson.

LECOMTE, R. (1991). Préface à R. MAYER R. et F. OUELLET, Méthodologie de recherche pour les intervenants sociaux. Boucherville: Gaëtan Morin éditeur.

LE POUlTiER, F. (1990). RECHERCHES évaluatives en travail social. Grenoble: Presses universitaires de Grenoble.

Les deux ans du RMI, Conseil des ministres du 12 décembre 1990.

LÉVI-STRAUSS, C. (1960). Le totémisme aujourd'hui. Paris.

LINTON, R. (1945 [1964]). Les fondements culturels de la personnalité. Paris: Dunod.

Makarenko, A. (1939). Poème pédagogique. Moscou : Éd. du Progrès.

MALINOWSKI, B. (1944 [1968]). Une théorie scientifique de la culture. Paris: Maspero.

March, J.G. et H.A. SIMON (1971). Les organisations. Paris : Dunod.

Mauss, M. (1933). Essai sur le don, Sociologie et anthropologie. Paris: PUF, 1968.

MAYER, R. et F. OUELLET (1991). Méthodologie de recherche pour les intervenants sociaux. Boucherville: Gaëtan Morin éditeur.

MEAD, G.H. (1933). L'esprit, le soi et la société. Paris : PUF.

MEHAN, H. (1978). "Structuring school structure», Harvard Educational Review, $48,1$.

MENDRAS, H. (1989). Éléments de sociologie. Paris: A. Colin.

MERTON, R.K. (1965). Éléments de théorie et de méthode sociologique. Paris : Plon.

MOYNIHAN, D.P. (1970). The maximum feasible misunderstanding. New York: Free Press.

MURY, G. (1972). "Note sur l'évolution du travail social», Esprit, mai.

OLSON, M. (1978). La logique de l'action collective. Paris: PUF.

PADIOLEAU, J.G. (1986). L'ordre social. Paris: L'Harmattan.

PALMADE, G. (1976 [1988]), Les méthodes en pédagogie. Paris: PUF. Que saisje? $13^{\mathrm{e}}$ éd.

Paugam, S. (1990). "Les statuts de la pauvreté assistée », Revue française de sociologie, 1.

Peters, T.J et R.H. WATERman (1983). Le prix de l'excellence. Paris : InterÉditions. PIAGET, J. (1965). Six études de psychologie. Paris: Gonthier.

PLATARD, Y. (1991). "L'Afrique trente ans après ", Revue des deux mondes, sept. POSTIC, M. et J.-M. De KeTELE (1988). Observer les situations éducatives. Paris: PUF.

RAWLS, J. (1987). Théorie de la justice. Paris: Seuil.

ROGERS, C.R. et R.F. DYMOND (1954). Psychotherapy and personality change. Chicago: University of Chicago Press.

ROGERS, C.R. (1965) Le développement de la personne. Paris: Dunod. 
ROGERS, C.R. (1979). Un manifeste personnaliste. Paris: Dunod.

SAINSAULIEU, R. (1977). L'identité au travail. Paris: FNSP.

SAINSAUlieu, R. (1987). Sociologie de l'organisation et de l'entreprise. Paris: FNSP.

SALBREUX (1979). "Déficience mentale et autonomie", Neuropsychiatrie de l'enfance, 27.

SCHUTZ, A. (1987). Le chercheur et le quotidien. Paris: Méridiens.

SÈVE, L. (1974). Marxisme et théorie de la personnalité. Paris : Éd. sociales.

SORMAN, G. (1987). La nouvelle richesse des nations. Paris: Fayard.

SURET-CANALE, J. (1972). "La théorie marxiste du développement des sociétés", dans CERM, Sur le mode de production asiatique. Paris: Éd. sociales.

TOMKIEWICZ, S. (1972). "Le foyer de semi-liberté de Vitry », La Psychiatrie de I'enfant, XVII, 2.

TOYNBEE, A.J. (1975). L'histoire : les grands mouvements de l'histoire. Paris : Elsevier.

Trottier, G. (1992). Conversation avec l'auteur. Sainte-Foy, Université Laval.

VERDÈS-LEROUX, J. (1978). Le travail social. Paris : Minuit.

WARREN, P. (1980). Studies in cross-cultural psychology. Londres: Academic Press.

WeBER, M. (1920 [1971]). Économie et société. Paris : Plon.

Weber, M. (1946). Essays in Sociology. New York: Oxford Univ. Press.

WILSON, O. (1970). L'humaine nature. Paris: Fayard.

WOLFENSBERGER, W. et L. GLENN (1975). Programme d'analyse des systèmes de service. Institut national de la déficience mentale, York University, Ont.

ZEROULOU, Z. (1988). "La réussite scolaire des enfants d'immigrés, I'apport d'une approche en termes de mobilisation ", Revue française de sociologie, XXIX, 447-470. 